\title{
Novel compound variants of the TMTC3 gene cause cobblestone lissencephaly-like syndrome: A case report
}

\author{
GUANGHUA LIU ${ }^{1}$, QING ZHOU ${ }^{1}, \mathrm{HAN} \mathrm{LIN}^{1}$, NIU LI ${ }^{2}, \mathrm{HONG}^{1}$ and JIAN WANG ${ }^{2}$ \\ ${ }^{1}$ Department of Pediatric Internal Medicine, Fujian Maternity and Child Health Hospital, Affiliated Hospital of Fujian \\ Medical University, Fuzhou, Fujian 350001; ${ }^{2}$ Department of Medical Genetics and Molecular Diagnostic Laboratory, \\ Shanghai Children's Medical Center, Shanghai Jiaotong University School of Medicine, Shanghai 200127, P.R. China
}

Received January 31, 2020; Accepted August 7, 2020

DOI: $10.3892 /$ etm.2020.9226

\begin{abstract}
Biallelic variants in the transmembrane O-mannosyltransferase targeting cadherins 3 (TMTC3) gene have been reported to cause two distinct types of neuron migration defect diseases, known as cobblestone lissencephaly (COB) and periventricular nodular heterotopia (PVNH), combined with intellectual disability and nocturnal seizures. The aim of the current study was to identify the genetic cause of a 22-month-old Chinese boy who presented with white matter plaques, a small frontal lobe, myelin dysplasia, microcephaly, psychomotor delay, language development delay, truncal hypotonia, intractable epilepsy, infantile spasm and bilateral single transverse palmar creases. Whole-exome sequencing revealed novel heterozygous variant compounds in the TMTC3 gene (c.1123G>A, p.Glu375Lys and c.1126_1129del, p.Arg376Tyrfs*13). Most of the clinical features of the patient are consistent with COB. However, the deformities in the brain (white matter plaques, small frontal lobe and myelin dysplasia) in the patient were more severe compared with those generally exhibited by PVNH, but less severe compared with those presented by COB. Moreover, the patient exhibited bilateral single transverse palmar creases, which, to the best of our knowledge, have not been described previously in patients with a TMTC3 variation. In summary, the current study reported
\end{abstract}

Correspondence to: Dr Hong Ye, Department of Pediatric Internal Medicine, Fujian Maternity and Child Health Hospital, Affiliated Hospital of Fujian Medical University, 18 Daoshan Road, Fuzhou, Fujian 350001, P.R. China

E-mail: yhfjsfy99@163.com

Professor Jian Wang, Department of Medical Genetics and Molecular Diagnostic Laboratory, Shanghai Children's Medical Center, Shanghai Jiaotong University School of Medicine, 1678 Dongfang Road, Shanghai 200127, P.R. China

E-mail: labwangjian@shsmu.edu.cn

Key words: cobblestone lissencephaly, periventricular nodular heterotopia, transmembrane O-mannosyltransferase targeting cadherins 3 , novel heterozygous variant compound, bilateral single transverse palmar creases a pediatric Chinese patient with COB-like syndrome caused by TMTC3 gene variations. The present results indicated that variation in the TMTC3 gene can lead to highly variable clinical phenotypes.

\section{Introduction}

The transmembrane O-mannosyltransferase targeting cadherins 3 (TMTC3) gene (Online Mendelian Inheritance in Man no. 617218) is located on chromosome 12q21.32 and encodes a type of O-mannosyltransferase comprised of 914 amino acids composed of nine transmembrane domains and 10 tetratricopeptide repeat (TPR) domains (data obtained from the UniProt database; uniprot.org/uniprot/Q6ZXV5). The most well-known function of the TMTC3 protein is its action as a positive regulator of the endoplasmic reticulum (ER) stress response by binding and interacting with protein disulphide-isomerase A3, which is an ER protein involved in the folding of glycoproteins and is overexpressed under conditions of ER stress such as the accumulation of misfolded proteins $(1,2)$.

Previous studies have reported that biallelic mutations of the TMTC3 gene result in two different neurologic defect syndromes in humans. The first one is cobblestone lissencephaly (COB), reported by Jerber et al (3) in 2016, which is mainly characterized by moderate to severe psychomotor delay, language development delay, intellectual disability (ID), truncal hypotonia, intractable seizure and malformations of the brain (agyria, ventriculomegaly, hypoplasia of the corpus callosum and hypoplasia and/or dysplasia of the brainstem and cerebellum). In addition, certain patients exhibit microcephaly, clubfoot and visual problems (3). The other syndrome was described by Farhan et al (4) in 2017, in which the patients were affected with periventricular nodular heterotopia (PVNH), ID and nocturnal seizures. Though there are certain overlapping clinical symptoms (ID and seizure) between the two syndromes, large phenotypic differences are observed, particularly for psychomotor and language development and the onset age of seizures (4). These findings prompted the current study to investigate an additional case to further examine the TMTC3 gene variation-related phenotype.

The current study described a novel compound heterozygous variant of the TMTC3 gene in a 22-month-old Chinese 
boy who, to a certain extent, exhibited a COB-like phenotype. The degree of brain deformity in the patient was between that characterized by COB and PVNH. Additionally, bilateral single transverse palmar creases as a novel phenotype due to TMTC3 variation were reported.

\section{Case report}

The patient was a 22-month-old Chinese boy [height, $88 \mathrm{~cm}$ (+0.2 SD); weight, $11.5 \mathrm{~kg}$ (-0.7 SD); head circumference, $45 \mathrm{~cm}(-2.3 \mathrm{SD})]$ who was the second child born at full term via cesarean section to physically healthy and nonconsanguineous Chinese parents. He was admitted to Department of Pediatric Internal Medicine of Fujian Maternity and Child Health Hospital (Fuzhou, China) for a comprehensive examination in March 2019. The patient presented with recurrent epileptic spasms at a frequency of 1-3 times/day for 2-3 days/week since he was 1 year old. Coronal magnetic resonance imaging from Department of Pediatrics, Nanping People's Hospital (Nanping, Fujian) revealed white matter plaques, a small frontal lobe and myelin dysplasia. Physical examination identified truncal hypotonia and bilateral single transverse palmar creases (Fig. 1A), and the electroencephalogram reported typical epileptoid discharge (Fig. 1B). The patient was diagnosed with intractable epilepsy and infantile spasm. The gross and fine motor development of the patient was significantly delayed compared with his peers. The patient could sit with support; however, he was unable to stand alone or speak. Infections by the Epstein-Barr virus or cytomegalovirus were ruled out. No abnormalities were reported using abdominal ultrasound or ophthalmic examination. The 12-year-old sister of the patient did not exhibit the aforementioned features.

The patient was suspected of having a genetic central nervous system syndrome. Therefore, whole-exome sequencing (WES) was performed to identify the genetic makeup of the patient, using a previously described experimental procedure, according to the manufacturer's protocols $(5,6)$. Briefly, a total of $3 \mu \mathrm{g}$ DNA from the patient was sheared to segments sized 150-200 bp using a Covarias ${ }^{\circledR}$ M220 Ultrasonicator system (Covaris, Inc.). DNA integrity was verified by electrophoresis on a $1 \%$ agarose gel. The adapter-ligated library was generated using the Agilent SureSelect Target Enrichment system (Agilent Technologies, Inc.) and the capture library, including both coding exons and flanking intronic regions, was produced with a SureSelect XT Human All Exon V6 reagent kit (cat. no. 5190-8863; Agilent Technologies, Inc.). Following this, clusters were generated via isothermal bridge amplification using an Illumina cBot station (Illumina, Inc.). Mass concentrations were measured with a Qubit dsDNA HS Assay kit (cat. no. Q32851; Thermo Fisher Scientific, Inc.). The library peak size was detected using the Bioanalyzer 2100 system (Agilent Technologies, Inc.). The average size value from the Bioanalyzer 2100 was used as the library size for conversion of mass concentration into molar concentration. Molar concentration $=(\mathrm{Ax} 1,000,000) /(\mathrm{Sx} 650)$, where $\mathrm{A}$ is the mass concentration (ng/ $\mu \mathrm{l})$ and $\mathrm{S}$ is the library size (bp). The final loading concentration was $0.7 \mathrm{nM}$. Paired-end sequencing with a read length of $150 \mathrm{bp}$ was performed using a NovaSeq 6000 S4 reagent kit (cat. no. 20012866; Illumina, Inc.) on an Illumina NovaSeq 6000 System (Illumina, Inc.).
After sequencing, the image files in binary base cell format were generated. CASAVA software (version no. 1.8; Illumina, Inc.) was used to perform base calling and demultiplexing to generate raw fastq files (primary analysis). Raw reads were trimmed using Skewer (version no. 0.2.2; https://sourceforge. net/projects/skewer/) to remove adapter sequences and low-quality reads. Subsequently, the trimmed data were aligned against a reference human genome (GRCh37/hg19; single nucleotide polymorphism; 153) using NextGENe ${ }^{\circledR}$ software (version no. 2.4.2; softgenetics.com/NextGENe_011. php; SoftGenetics, LLC). All single nucleotide variants and indels were presented in variant cell format and were uploaded to Ingenuity ${ }^{\circledR}$ Variant Analysis ${ }^{\mathrm{TM}}$ (version no. 2.11; qiagenbioinformatics.com/products/ingenuity-variant-analysis; Qiagen, Inc.) for bioinformatics analysis and interpretation. WES raw data was deposited into the Mendeley Data online database (dx.doi.org/10.17632/69j4nzggdx.1).

Common variants with allele frequencies (AF) $>1 \%$ in the gnom AD database (version no. 2.1.1; gnomad.broadinstitute. org) and benign variants, including synonymous, harmless missense variants predicted using PolyPhen-2 (version no. 2.2; genetics.bwh.harvard.edu/pph2/) and MutationTaster (https://mutationtaster.org/) (7) software and those predicted to have no impact on splicing using MaxEntScan software (hollywood.mit.edu/burgelab/maxent/Xmaxentscan_scoreseq_acc. html) (8), were initially excluded. Subsequently, clinical symptoms of global developmental delay and epilepsy served as filtering indexes to analyze candidate variants. Finally, a compound heterozygous variant in the TMTC3 gene was identified in the patient (Fig. S1). In total, one was a missense variant with an extremely low AF (0.00042\%; gnomAD database; GenBank accession no. NM_181783.3) in exon 8 that generates an amino acid conversion (c.1123G $>A$, p.Glu375Lys; rs750602559). The other was a deletion of four bases (c.1126_1129delCGAG) in exon 8, which was absent in the gnomAD database and was predicted to lead to a frameshift mutation resulting in a premature stop codon (p.Arg376Tyrfs*13). To the best of our knowledge, neither variant has been previously reported.

The identified TMTC3 variants were confirmed in the patient and his parents using Sanger sequencing (Fig. 2A and B). The primers for amplification of the TMTC3 gene were designed using Prime 3 online software (version no. 4.1.0; primer3.ut.ee). The primers designed for exon 8 were as follows: Forward, 5'-GGATTCAAGTATCAGATGCCCA-3' and reverse, 5'-AGT AGGTGCCATGGAGCTTT-3'. Both exons and exon-intron boundaries were amplified by PCR. The reaction mixture for each amplification contained 1X Premix Taq (Ex Taq ${ }^{\mathrm{TM}}$ version 2.0; cat. no. RR003; Takara Biotechnology Co., Ltd.), $100 \mathrm{ng}$ genomic DNA and 1 pmol forward and reverse primers to a final volume of $25 \mu \mathrm{l}$. The reaction was performed under the following PCR conditions: Initial denaturation at $95^{\circ} \mathrm{C}$ for $5 \mathrm{~min}$, followed by 19 cycles of $95^{\circ} \mathrm{C}$ for $30 \mathrm{sec}, 65^{\circ} \mathrm{C}$ for $30 \mathrm{sec}$ and $72^{\circ} \mathrm{C}$ for $45 \mathrm{sec} ; 14$ cycles of $95^{\circ} \mathrm{C}$ for $30 \mathrm{sec}$, $55^{\circ} \mathrm{C}$ for $30 \mathrm{sec}$ and $72^{\circ} \mathrm{C}$ for $45 \mathrm{sec}$; and a final elongation step at $72^{\circ} \mathrm{C}$ for $5 \mathrm{~min}$ using a C1000TM Thermal Cycler (Bio-Rad Laboratories, Inc.). PCR products were separated by $1 \%$ agarose gel (Sangon Biotech Co., Ltd.) with SYBR ${ }^{\mathrm{TM}}$ Safe DNA Gel Stain (cat. no. S33102; Thermo Fisher Scientific; Inc.) and then purified using a QIAquick Gel Extraction kit 
A

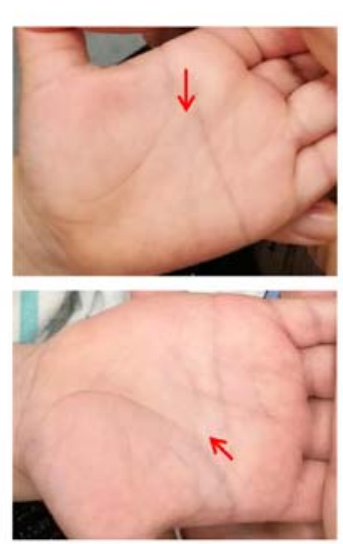

B

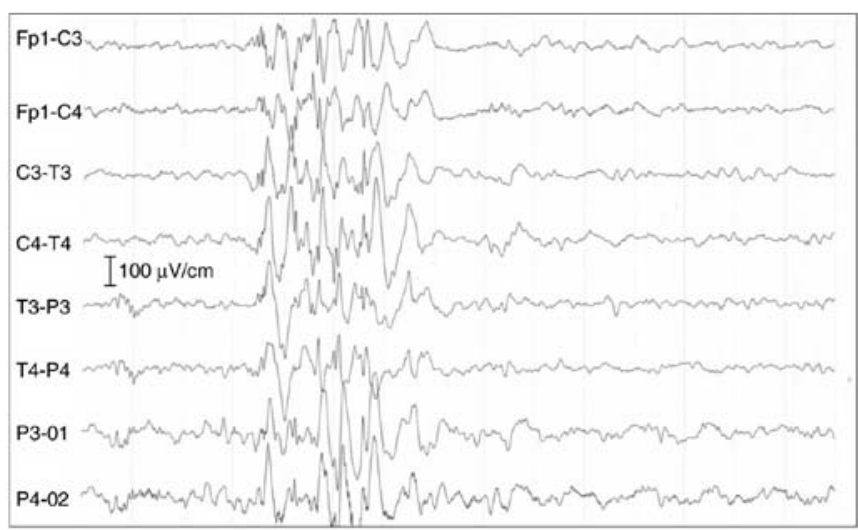

Figure 1. Clinical features of the patient. (A) Bilateral single transverse palmar creases. Red arrows indicate the missing transverse palmar crease. (B) Electroencephalogram examination results.

A

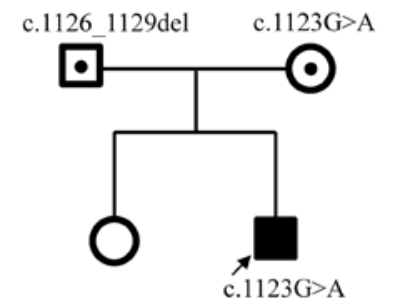

c.1126_1129delCGAG

C

Homo sapiens
Drosophila melanogas
Mus musculus
Sus scrofa
Macaca mulatta
Ovis aries
Felis catus
Canis lupus familiar

B
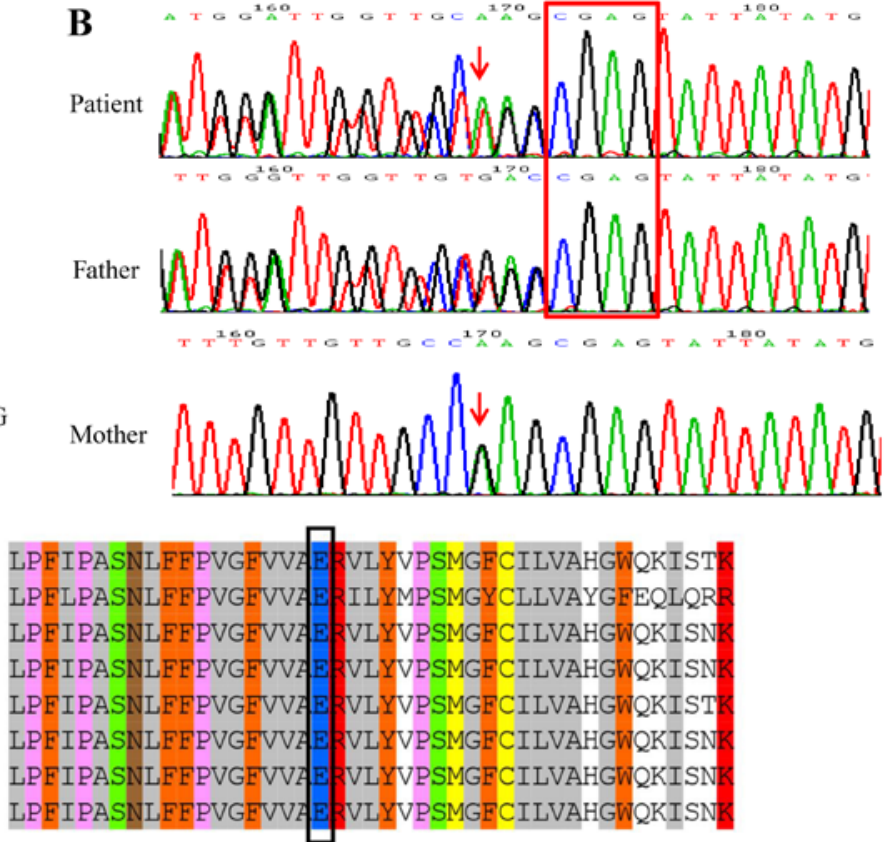

Figure 2. Genetic sequencing of the patient. (A) Pedigree of the patient. (B) Sanger sequencing using the reverse primer confirmed that the patient harbored compound heterozygous variants of c.1123G $>$ A and c.1126_1129delCGAG in exon 8, which were inherited from the mother (red arrow) and father (red rectangle), respectively. c.1123 is 'AT' due to both variants exhibited by the patient. (C) Phylogenetic comparison of the transmembrane O-mannosyltransferase targeting cadherins 3 protein among various species. The position of Glu375 within a highly conserved region is indicated by a black box. c, chromosome.

(Qiagen $\mathrm{GmbH}$ ). The purified DNA was sequenced using an ABI3730XL sequencer (Applied Biosystems; Thermo Fisher Scientific, Inc.) with reverse primers. The sequence data were analyzed with Mutation Surveyor DNA Variant Analysis software (version no. 4.0.4; SoftGenetics, LLC). Sanger sequencing revealed that the missense variant was inherited from the mother of the patient and the frameshift variant from his father. Additionally, copy number variation (CNV) analysis was performed by comparing the sequence depth with the WES data from the other 20 samples of the same batch, using the NextGENe ${ }^{\circledR}$ software (SoftGenetics LLC). No clinically significant $\mathrm{CNVs}$ were found.

After sequencing, several types of in silico tools were applied to assess the pathogenicity of the Glu375Lys variant.
The functional prediction of the identified variant was analyzed with MultAlin online software (multalin.toulouse.inra. fr/multalin) (9), CADD online software (version no. 1.6; cadd. gs.washington.edu), PROVEAN (version no. 1.1; provean.jcvi. org), MutationTaster online software and PolyPhen-2 online software (version no. 2; genetics.bwh.harvard.edu/pph2).

In silico analysis from the MultAlin online software revealed that the Glu375 amino acid residue of TMTC3 was highly conserved in multiple species (Fig. 2C). Functional prediction of the Glu375Lys variant demonstrated a harmful effect on the TMTC3 protein resulting from PolyPhen-2 (probably damaging; score, 1), PROVEAN (damaging; score, -3.9), MutationTaster (disease causing; score, 1) and CADD (damaging; score, 34). 
Table I. Phenotypic comparison of the patient in the current study and previously reported patients.

\begin{tabular}{|c|c|c|c|}
\hline Clinical feature & $\begin{array}{l}\text { Patients with COB }(\mathrm{n}=9) \\
\text { reported by Jerber et al }(3)\end{array}$ & $\begin{array}{l}\text { Patients with PVNH }(n=4) \\
\text { reported by Farhan et al }(4)\end{array}$ & $\begin{array}{l}\text { Patient }(n=1) \text { in } \\
\text { the current study }\end{array}$ \\
\hline \multicolumn{4}{|l|}{ Psychomotor development } \\
\hline Motor skills & Delayed (9/9) & Normal & Delayed \\
\hline Language & Delayed (5/9), absent (4/9) & Delayed (1/4) & Delayed \\
\hline \multicolumn{4}{|l|}{ Seizures } \\
\hline Type & $\begin{array}{c}\text { Intractable and } \\
\text { infantile-onset epilepsy }\end{array}$ & Nocturnal seizures & $\begin{array}{l}\text { Intractable and } \\
\text { infantile-onset epilepsy }\end{array}$ \\
\hline Age of onset & 4-8 months & $2-5$ years & 1 year \\
\hline Frequency & Daily to weekly & $\begin{array}{l}\leq 4 \text { times/night, } \\
\leq 4-5 \text { days/week }\end{array}$ & $\begin{array}{l}\leq 3 \text { times/day, } \\
\leq 2-3 \text { days a week }\end{array}$ \\
\hline \multicolumn{4}{|l|}{ Neurological abnormalities } \\
\hline Hypotonia & $9 / 9$ & None & Yes \\
\hline Intellectual disability & $9 / 9$ & $4 / 4$ & $\begin{array}{l}\text { Too young for } \\
\text { assessment }\end{array}$ \\
\hline Microcephaly & $3 / 7$ & None & Yes \\
\hline \multicolumn{4}{|l|}{ MRI findings } \\
\hline $\mathrm{COB}$ & $7 / 9$ & None & $\begin{array}{l}\text { White matter plaques, } \\
\text { small frontal lobe and } \\
\text { myelin dysplasia }\end{array}$ \\
\hline Ventriculomegaly & $7 / 9$ & None & \\
\hline Corpus callosum hypoplasia & $5 / 9$ & None & \\
\hline Brainstem hypoplasia & $6 / 9$ & None & \\
\hline Cerebellum hypoplasia & $6 / 9$ & None & \\
\hline Encephalocele & $2 / 9$ & None & \\
\hline Bilateral periventricular heterotopias & None & $3 / 4$ & \\
\hline
\end{tabular}

COB, cobblestone lissencephaly; PVNH, periventricular nodular heterotopia.

\section{Discussion}

TMTC3 belongs to a putative family of O-mannosyltransferases consisting of four TPR-containing proteins (TMTC1-TMTC4) (3). TPR domains are critical for protein-protein interactions involved in various biological processes, including biomineralization, synaptic vesicle fusion, protein folding, organelle targeting and protein import $(10,11)$. The human TMTC3 protein was initially identified in the context of renal transplant surgeries and was revealed to be upregulated in the blood of operationally tolerant individuals (12). In addition to its role in the ER stress response, TMTC3 was recently found to contribute to the O-mannosylation of E-cadherin, which is crucial for E-cadherin-mediated cell-cell adhesion (13-15). Furthermore, mSmile, the murine homolog of TMTC3, is necessary for bronchial smooth muscle and alveolar myofibroblast development, and deficiency results in early neonatal lethality in mice due to airway branching morphogenesis defects during fetal lung development and alveolarization defects after birth (16).

$\mathrm{COB}$ is a severe brain malformation resulting from the overmigration of neuronal cells, whereas PVNH is characterized as a common brain malformation due to neurons failing to migrate from the ventricles (4). However, the role of TMTC3 in the development of the nervous system is poorly understood. Farhan et al (4) revealed that TMTC3 is localized at presynaptic terminals in rat brains via colocalization with the vesicular $\gamma$ aminobutyric acid transporter. Specific knockdown of Drosophila neuronal TMTC3 has been reported to cause seizure susceptibility, which can be recovered by human TMTC3 (13). Furthermore, knockout of the TMTC3 gene in 293 cells was previously reported to lead to cellular adhesion defects via markedly reduced binding to the extracellular region of E-cadherin, which was also hypothesized to be a possible molecular mechanism for neuron migration defects (14). While the present study hypothesized that TMTC3 may serve a role in the development of the nervous system, the precise molecular mechanisms are yet to be fully elucidated. Further studies should consider using animal models with a brain tissue-specific TMTC3 knockout.

The current study presented a male Chinese patient who exhibited white matter plaques, a small frontal lobe, myelin dysplasia, microcephaly, psychomotor delay, language development delay, truncal hypotonia, intractable epilepsy, infantile spasm and bilateral single transverse palmar creases. DNA sequencing demonstrated that the patient harbored compound heterozygous variants for c.1123G $>A$ 


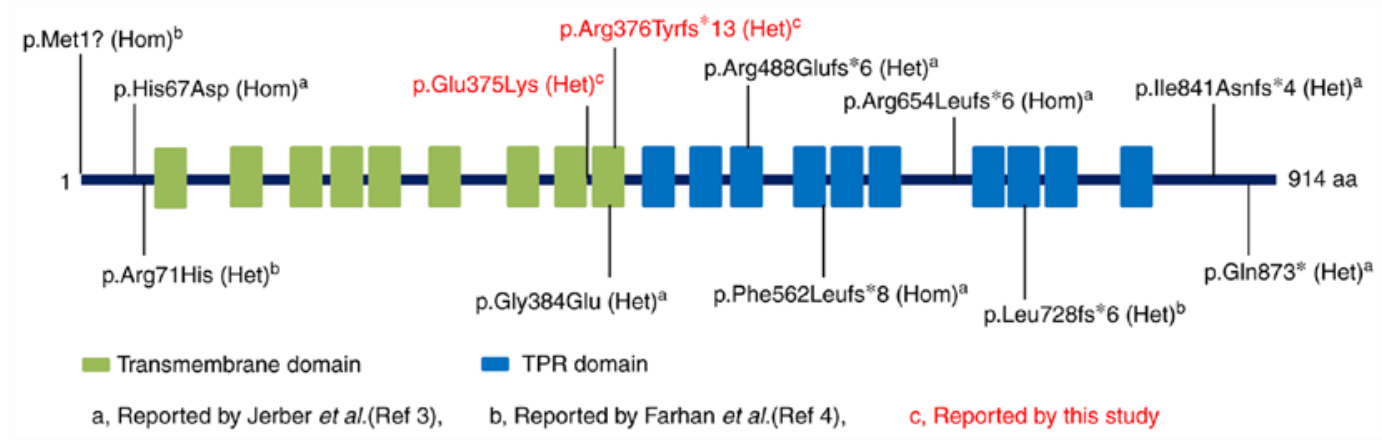

Figure 3. Schematic diagram of the distribution of reported variants in the transmembrane O-mannosyltransferase targeting cadherins 3 gene. In total, 12 pathogenic variants from two previously published articles and the present study were distributed throughout the TMTC 3 protein, including eight null variants (six frameshift, one nonsense, and one initial codon variant) and four missense variants. TPR, tetratricopeptide repeat.

(p.Glu375Lys) and c.1126_1129delCGAG (p.Arg376Tyrfs*13) in the TMTC3 gene. According to the guidelines developed by the American College of Medical Genetics and Genomics/Association for Molecular Pathology variant-interpretation (17), the p.Arg376Tyrfs*13 variant is classified as pathogenic (PVS1+PM2+PP4) and the p.Glu375Lys variant is likely pathogenic $(\mathrm{PM} 2+\mathrm{PM} 3+\mathrm{PP} 3+\mathrm{PP} 4)$. Most of the characteristics of the patient were consistent with $\mathrm{COB}$, including microcephaly, psychomotor delay, language development delay, truncal hypotonia, intractable epilepsy and infantile spasm (Table I). However, the brain deformities presented in the patient were more severe compared with those generally exhibited by PVNH, but less severe compared with those presented by COB. Moreover, to the best of our knowledge, the bilateral single transverse palmar creases in the patient have not been described in previously reported patients, indicating that this may be a novel phenotype resulting from TMTC3 variation. The patient was diagnosed with TMTC3 variation-related COB-like syndrome by comparing the phenotype with two previously reported groups of patients $(3,4)$.

To date, a total of 10 variants of the TMTC 3 gene have been reported by two groups. Jerber et al (3) described four homozygous (p.Met1?, p.His67Asp, p.Arg654Leufs*6 and p.Phe562Leufs*8) and two compound heterozygous variants (p.Arg488Glufs*6/p.Gln873* and p.Gly384Glu/p. Ile841Asnfs*4) in six patients with COB. Farhan et al (4) identified a compound heterozygous variant (p.Arg71His/p. Leu728fs*6) from four siblings in a pedigree where the patients had PVNH with nocturnal seizures and ID. Unlike patients with COB, patients with PVNH tended to have normal psychomotor and language development, less severe brain lesions and a much later onset age of seizures (Table I). However, in addition to the two variants, the 12 variants, including four missense and eight null variants, were evenly distributed on the TMTC3 protein (Fig. 3). It is difficult to compare the phenotypic relationship of the two types of diseases according to the variant distribution, or the variant type or composition. Functionally, knockdown of TMTC3 has been reported to result in delayed gastrulation in Xenopus laevis, which may be rescued by complementation of wild-type TMTC 3 . The three disease-causing missense variants (p.His67Asp, p.Arg71His and p.Gly384Glu) failed to rescue the delayed gastrulation phenotype, further supporting the deleterious impact on the TMTC3 protein (14). The in vitro study using mutant plasmids revealed that the PVNH-related TMTC3 mutant protein (p.Arg71His) had a similar half-life compared with wild-type TMTC3, whereas COB-associated TMTC3 variants (p.Gly384Glu, p.Arg488Glufs*6 and p.Phe562Leufs*8) led to significantly reduced half-lives (14). This increased instability may be an explanation for the severe phenotype of patients with COB. However, further functional investigations should be performed to directly study how the variants affect the development of the nervous system, as no half-life alterations were observed from the other four COB-associated TMTC3 variants (p.His67Asp p.Arg654Leufs*6 p.Ile841Asnfs*4 p.Gln873*) (14).

In conclusion, the current study identified a novel compound heterozygous variant in the TMTC 3 gene that caused severe neurological defects in a pediatric Chinese patient. The results further support the observation that variation in the TMTC3 gene leads to a recessive $\mathrm{COB}$ phenotype. Moreover, to the best of our knowledge, the current study was the first to indicate the feature of bilateral single transverse palmar creases in patients with TMTC3 variation.

\section{Acknowledgements}

Not applicable.

\section{Funding}

The current study was supported by the Guiding Project of Fujian Province Health Commission (grant no. 2019Y0057) and the Project of Science and Technology Innovation, Fujian Province Health Commission [grant no. 2017(804)].

\section{Availability of data and materials}

The WES raw data (named 'VCF file of WES data for a TMTC3 variation patient') is available from the Mendeley Data online database (http://dx.doi.org/10.17632/69j4nzggdx.1).

\section{Authors' contributions}

GL, HY and JW conceptualized and designed the current study, drafted the initial manuscript and reviewed and revised 
the manuscript. NL and JW were responsible for the genetic diagnosis and interpretation of genetic findings. QZ and HL were responsible for patient treatment and medical history data collection. The authors agreed to be accountable for all aspects of the current work. All authors read and approved the final manuscript.

\section{Ethics approval and consent to participate}

All procedures followed were in accordance with the ethical standards of the Fujian Maternity and Child Health Hospital (Fuzhou, China) on human experimentation and with the Helsinki Declaration of 1975 (revised in 2000), and the protocol was approved by the Ethics Committee of Fujian Maternity and Child Health Hospital, Fuzhou, China (approval no. FMCHHIRB-2019019). The patient was enrolled from the Fujian Maternity and Child Health Hospital, and written informed consent was obtained from the patient's family.

\section{Patient consent for publication}

Written informed consent was obtained from the patient's family.

\section{Competing interests}

The authors declare that they have no competing interests.

\section{References}

1. Racapé M, Duong Van Huyen JP, Danger R, Giral M, Bleicher F, Foucher Y, Pallier A, Pilet P, Tafelmeyer P, Ashton-Chess J, et al: The involvement of SMILE/TMTC3 in endoplasmic reticulum stress response. PLoS One 6: e19321, 2011.

2. Parakh S and Atkin JD: Novel roles for protein disulphide isomerase in disease states: A double edged sword? Front Cell Dev Biol 3: 30, 2015.

3. Jerber J, Zaki MS, Al-Aama JY, Rosti RO, Ben-Omran T, Dikoglu E, Silhavy JL, Caglar C, Musaev D, Albrecht B, et al: Biallelic mutations in TMTC3, encoding a transmembrane and TPR-containing protein, lead to cobblestone lissencephaly. Am J Hum Genet 99: 1181-1189, 2016.

4. Farhan SMK, Nixon KCJ, Everest M, Edwards TN, Long S, Segal D, Knip MJ, Arts HH, Chakrabarti R, Wang J, et al: Identification of a novel synaptic protein, TMTC3, involved in periventricular nodular heterotopia with intellectual disability and epilepsy. Hum Mol Genet 26: 4278-4289, 2017.
5. Yu T, Li J, Li N, Liu R, Ding Y, Chang G, Chen Y, Shen Y, Wang $X$ and Wang J: Obesity and developmental delay in a patient with uniparental disomy of chromosome 2 . Int J Obes (Lond) 40: 1935-1941, 2016.

6. Li N, Xu Y, Zhang Y, Li G, Yu T, Yao R, Zhou Y, Shen Y, Yin L, Wang $X$ and Wang J: Biallelic ERBB3 loss-of-function variants are associated with a novel multisystem syndrome without congenital contracture. Orphanet J Rare Dis 14: 265, 2019.

7. Schwarz JM, Cooper DN, Schuelke M and Seelow D: MutationTaster2: Mutation prediction for the deep-sequencing age. Nat Methods 11: 361-362, 2014.

8. Yeo $\mathrm{G}$ and Burge CB: Maximum entropy modeling of short sequence motifs with applications to RNA splicing signals. J Comput Biol 11: 377-394, 2004.

9. Corpet F: Multiple sequence alignment with hierarchical clustering. Nucleic Acids Res 16: 10881-10890, 1988.

10. D'Andrea LD and Regan L: TPR proteins: The versatile helix. Trends Biochem Sci 28: 655-662, 2003.

11. Zeytuni $\mathrm{N}$ and Zarivach R: Structural and functional discussion of the tetra-trico-peptide repeat, a protein interaction module. Structure 20: 397-405, 2012.

12. Brouard S, Mansfield E, Braud C, Li L, Giral M, Hsieh SC, Baeten D, Zhang M, Ashton-Chess J, Braudeau C, et al: Identification of a peripheral blood transcriptional biomarker panel associated with operational renal allograft tolerance. Proc Natl Acad Sci USA 104: 15448-15453, 2007.

13. Larsen ISB, Narimatsu Y, Joshi HJ, Siukstaite L, Harrison OJ, Brasch J, Goodman KM, Hansen L, Shapiro L, Honig B, et al: Discovery of an O-mannosylation pathway selectively serving cadherins and protocadherins. Proc Natl Acad Sci USA 114: 11163-11168, 2017.

14. Graham JB, Sunryd JC, Mathavan K, Weir E, Larsen ISB, Halim A, Clausen H, Cousin H, Alfandari D and Hebert DN: ER transmembrane protein TMTC3 contributes to O-mannosylation of E-cadherin, Cellular Adherence and Embryonic Gastrulation. Mol Biol Cell 31: 167-183, 2020.

15. Larsen ISB, Narimatsu Y, Clausen H, Joshi HJ and Halim A: Multiple distinct O-Mannosylation pathways in eukaryotes. Curr Opin Struct Biol 56: 171-178, 2019.

16. Yun EJ and Vu TH: mSmile is necessary for bronchial smooth muscle and alveolar myofibroblast development. Anat Rec (Hoboken) 295: 167-176, 2012.

17. Richards S, Aziz N, Bale S, Bick D, Das S, Gastier-Foster J, Grody WW, Hegde M, Lyon E, Spector E, et al: Standards and guidelines for the interpretation of sequence variants: A joint consensus recommendation of the American college of medical genetics and genomics and the association for molecular pathology. Genet Med 17: 405-424, 2015.

This work is licensed under a Creative Commons Attribution-NonCommercial-NoDerivatives 4.0 International (CC BY-NC-ND 4.0) License. 